\title{
Investigating the Relationship Between Fun at Work and Citizenship Behavior with The Mediating Role of Organizational Learning (Case Study of Physical Education Teachers)
}

\author{
Mohammad Saeid Kiani ${ }^{1 *}$ and Shahab Bahrami ${ }^{2}$ \\ ${ }^{1}$ PhD Student, Department of Sports Management, Kermanshah Branch, Islamic Azad University, Kermanshah, Iran \\ ${ }^{2}$ Assistant Professor, Department of Sports Management, Kermanshah Branch, Islamic Azad University, Kermanshah, Iran
}

*Corresponding author: Mohammad Saeid Kiani, PhD Student, Department of Sports Management, Kermanshah Branch, Islamic

Azad University, Kermanshah, Iran

\begin{abstract}
ARTICLE INFO
Received: 幽 December 22, 2020

Published: 幽 January 06, 2021

Citation: Mohammad Saeid Kiani, Shahab Bahrami. Investigating the Relationship Between Fun at Work and Citizenship Behavior with The Mediating Role of Organizational Learning (Case Study of Physical Education Teachers). Biomed J Sci \& Tech Res 33(1)-2021. BJSTR. MS.ID.005332.
\end{abstract}

Keywords: Fun at Work; Citizenship Behavior; Organizational Learning; Physical Education Teachers

\section{ABSTRACT}

The purpose of this study was to investigate the relationship between fun at work and organizational citizenship behavior with the mediating role of organizational learning in physical education teachers in Kermanshah province. The statistical population of the study consisted of all staff (240 persons) of physical education teachers in Kermanshah province. 173 physical education teachers were studied through randomclustered sampling. The research instrument included the Organizational Citizenship Behavior Questionnaire developed by Podsakov and Mackenzie (1990), Workplace fun questionnaire developed by McDowell's (2004) and Neife's Organizational Learning Questionnaire (2001). Structural equation modeling by applying Smart PLS was conducted for data analysis. The results of this study showed that:

a) there was a significant relationship between "fun at work" and "organizational citizenship behavior"

b) there was a significant relationship between "fun at work" and "organizational learning", and

c) there was a significant relationship between "organizational learning" and "organizational citizenship behavior". Moreover, it was found that "organizational learning" has a partial mediating role on the relationship of "fun at work" and "citizenship behavior".

\section{Introduction}

The concept of organizational citizenship behavior was first introduced to the world of science by Batman and Organ in the early 1980s. Early research into organizational citizenship behavior was more about identifying responsibilities or behaviors that employees had in the organization but were often overlooked. Although these behaviors were incompletely measured and sometimes neglected in traditional evaluations of job performance, they were effective in improving organizational effectiveness [1]. There are three main types of research on organizational citizenship behavior. A series of studies have focused on predicting and empirically testing the factors that cause organizational citizenship behavior. In this regard, factors such as job satisfaction Ahmadi et al. [2,3], organizational commitment Baharifar et al. [4], organizational identity [5], organizational justice [6], trust [7], types of leadership and the relationship between leader and follower [8] and Have been proposed as factors that cause organizational citizenship behavior. On the other hand, a series of studies focus on the consequences of organizational citizenship behavior. In this regard, factors such as organizational performance, organizational effectiveness, organizational success, customer satisfaction, customer loyalty, social capital, etc. have been proposed. 
Few groups of research have focused exclusively on the concept of organizational citizenship behavior and have tried to redefine organizational citizenship behavior, determine its dimensions, or use standard analysis to create standard scales to measure this concept $[9,10]$. believe; Organizational citizenship behavior is a behavior based on personal discretion that is not among the job requirements of the employee, but also increases organizational effectiveness to satisfy the interests of stakeholders [11]. In fact, organizational citizenship behavior causes employees to prefer group and organizational interests over individual interests. In order to ensure organizational efficiency, organizations need the participation, generosity, sacrifice and self-sacrifice, and sometimes additional efforts of employees. Therefore, the voluntary work of employees is very important for organizations [12]. The structure (concept) of organizational citizenship behavior has several dimensions. Some researchers, including [13] have outlined five dimensions for organizational citizenship behavior. These dimensions include altruism, work conscience, civic virtue, politeness and kindness, and chivalry. Oregon has articulated the implications of these dimensions:

1. Altruism refers to the way in which employees help others;

2. Civic virtue, expressing responsible participation Employees are in the life cycle of the organization.

3. Respect and honor means that employees treat others with respect. The results of the studies of Katz and Kahn (1996) indicate that an organization needs the commitment of its employees to innovate, perform spontaneous behaviors and exceed the expectations of their role in order for their activities to be effective. Considering the positive consequences of organizational citizenship behavior such as organizational effectiveness, efficiency and organizational productivity, the existence of this behavior is desirable and important for all organizations. They seek to provide a suitable environment and use the factors influencing organizational citizenship behavior [14].

In this regard, research on the factors that lead to the creation and increase of organizational citizenship behavior, has examined various variables. For example, understanding organizational support and justice [15], job satisfaction Shiri Levy et al. [16], increasing social exchanges Amala et al. [17], leadership style Azma et al. [18] and organizational culture [19]. One of the factors that can affect organizational citizenship behavior is refreshment at work, which has been limited in previous research. [20] examined the direct and indirect impact of work refreshment on organizational citizenship behavior. According to the resource-demand model, refreshment at work is considered as a positive job source due to its social and interactive nature and leads to the well-being and emotional health of employees [20], on the other hand, According to the theory of social exchange, when employees benefit from the actions and activities of the organization, they consider themselves committed and obligated to show useful behaviors such as organizational citizenship behavior, Customer-centered behaviors, that ultimately improve the performance of the organization [21]. Given that people today spend a large part of their daily lives in the organization or workplace. Workplace stress causes employees to spend more energy than renewed energy.

Therefore, creating a refreshing work environment that creates positive emotions such as feelings of happiness in employees and has positive consequences such as organizational citizenship behavior, is one of the necessities of organizations that seek to increase productivity and improve their performance. . Fun at work refers to any work, interpersonal and social activity in the workplace that is joyful in nature and entertains and entertains people. According to Carl (2007) model, the dimensions of attitudes toward refreshment and experienced refreshment were considered as refreshment dimensions. Experience refreshment refers to the extent to which employees perceive the existence of refreshment in the workplace and attitudes In terms of recreation, the elements related to the importance and appropriateness of recreation at work as well as the perceived consequences of recreation are included. [20] has also examined these four dimensions in his research. These dimensions are: dimension of social interactions, rituals and festivals, individual freedom, general atmosphere. The dimension of social interaction refers to friendly mutual relations with colleagues. The dimension of rituals and festivals in the workplace refers to holding rituals and festivals and parties on various occasions in the workplace, the dimension of individual freedoms is related to independence and flexibility in dressing, listening to music and other dimensions of work. The overall atmosphere refers to the support of managers for fun and also the general feeling of fun at work. In addition, various studies have pointed to the positive consequences of refreshing work. Michael et al. [22] found in their research that refreshment at work improves job performance.

Plaster and Hatchi Sun (2016) bring refreshment to the work of refreshing and refreshing employees, which in turn increases their work ethic. [23] also found that refreshment at work has a significant effect on organizational commitment and job satisfaction of nurses. At the same time, some researchers have suggested that organizational refreshment affects organizational citizenship behavior. Rahimnia and Rahnama (2017) found in their research that there is a significant relationship between refreshment in work and organizational citizenship behavior. Geon Sik et al. [20] also found that refreshment at work has a direct and indirect effect on organizational citizenship behavior. However, more research is needed to understand the different contexts of this relationship and the role of mediators in this relationship. However, having fun at work through social interactions and participating in celebrations and occasions with co-workers doubles the chances of employees 
getting to know each other better, thus making them more likely to engage in social exchanges such as helping. The more partners participate, the more. Therefore, it can be inferred that refreshment at work improves the organizational learning process by increasing the social interactions of individuals, and this organizational learning provides the basis for improving organizational citizenship behavior. Organizational learning is achieved by sharing the insights, knowledge, experience and mental patterns of members of the organization. In fact, individuals and groups are the factors through which organizational learning is achieved. Argriss (1999) also considers organizational learning to be the sharing of knowledge, beliefs and assumptions among team members.

The scales used in this learning attempt to assess individuals' perceptions of knowledge storage, culture, vision, and organizational strategies and systems. Pietro et al. [24] They point to the role of social capital in group cohesion and the impact it has on organizational learning. They state that social capital among the members of the group causes the formation of the personality of the group and promotes cohesion and integration among the members, which promotes mutual help and organizational citizenship behaviors among the members. A group with high social capital flows data and information among themselves faster and the flow of knowledge and experiences of members as a result of continuous and stable communication based on trust turns tacit knowledge into explicit knowledge and facilitates knowledge transfer and learning promotion. Becomes organized Pietro et al. [25]. At the same time, Michael et al. [26] in their research found that refreshing in the organization increases learning in the organization. Anders et al. [27] in their research entitled the role of happiness and refreshing work on organizational citizenship behavior and ability Learning in Health Center Employees They found that happiness and wellbeing can improve organizational citizenship behavior and the ability of employees to learn.

However, the extent of organizational learning intervention as a mediating intervention in the relationship between work refreshment and organizational citizenship behavior in the sports organization in which job activity is very high and time consuming and is associated with employee burnout, has not yet been studied. And the need to establish refreshing processes in the organization requires extensive research support, which is felt in the research gap. Therefore, in this study we intend to propose a model including the relationship between fun at work and organizational citizenship behavior with the mediating role of organizational learning among physical education teachers in Kermanshah province Figure 1.

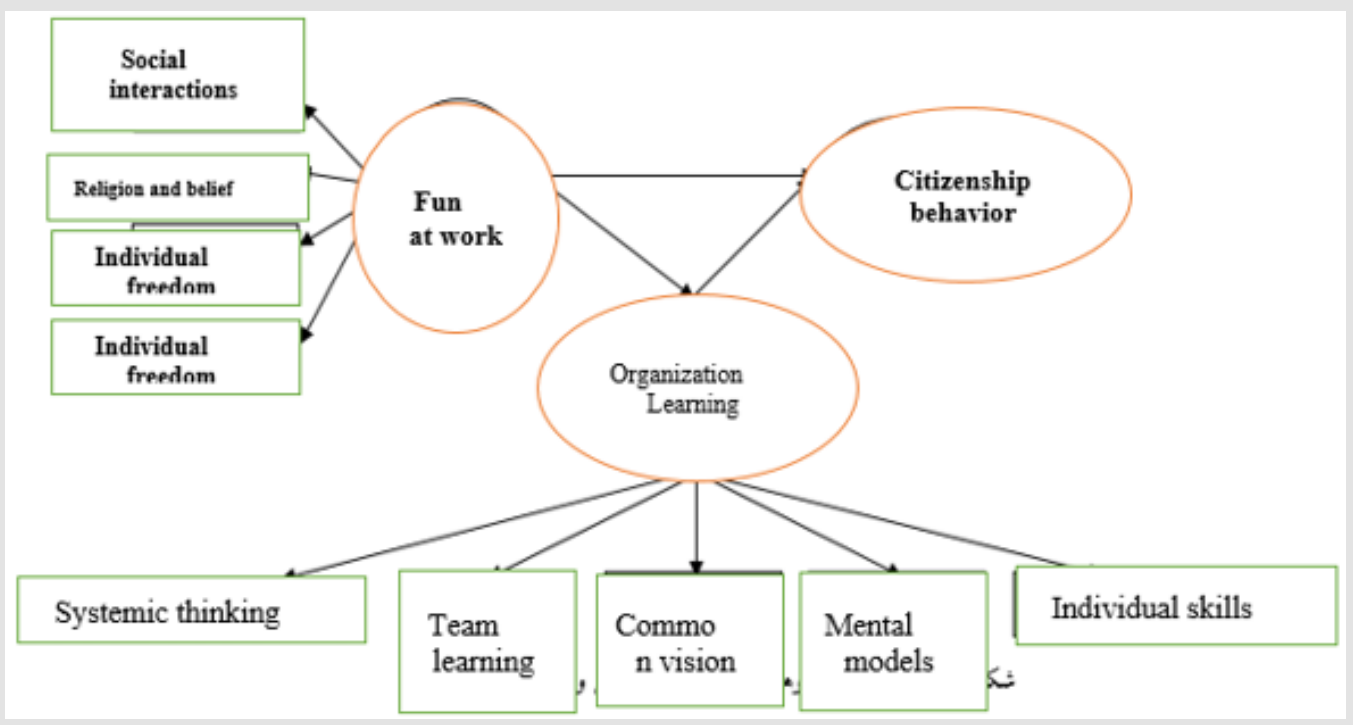

Figure 1: Conceptual model taken from Andalus et al. 2017.

\section{Methodology}

This field study was a descriptive and practical one. The statistical population of the study included all physical education teachers in Kermanshah province. The sampling method was random-cluster. In this way, Kermanshah province is divided into five clusters: North (Songhar, Paveh, Ravansar), South (IslamabadWest-Gilan-Gharb, Dalahou), West (Sarpolzahab, Qasr Shirin, Thalas Baba Jani), East (Kangavar, Sahneh, Hersin, Bistoon) and Markazi (Kermanshah) and one city was randomly selected from each cluster (Songhar, Islamabad Gharb, Qasr Shirin, Harsin and Kermanshah) and Statistical samples were randomly selected from each city according to Morgan table. Finally, 173 subjects were selected as a sample and all questionnaires were distributed and collected.

To collect data in this study, three Questionnaires have been used:

A) Padsakoff \& Mackenzie (1990) Organizational Citizenship Behavior Questionnaire: This questionnaire, which consists of 
24 questions and has the components of conscience (questions 1 to 5), chivalry (questions 6 to 10), virtue Civic (questions 11 to 14 ), humility (questions 15 to 19 ) and altruism (questions 20 to 24 ) are designed as five Likert options.

B) McDowell (2004) fun at work Questionnaire: In this study, four dimensions of social interaction, rituals and festivals, individual freedom and the general atmosphere dimension have been considered to measure the dimensions of refreshment in work. These dimensions include 18 items and are taken from McDowell's (2004) research. The dimension of social interactions includes six items, the dimension of rituals and feasts includes 4 items, the dimension of individual freedom includes 4 items and the social dimension includes six items, the dimension of rituals and holidays includes 4 items, the dimension of individual freedom includes 4 items and the general dimension consists of 5 items.

C) Nice Organizational Learning Questionnaire (2001): This questionnaire includes 24 questions and components of individual skills, mental models, shared vision Figure 2, team learning, and systemic thinking. This questionnaire is based on the Likert five-point scale. These questionnaires, Are standard questionnaires that have been used many times in various researches and its reliability has been confirmed. Reliability and validity in PLS are measured in two parts:

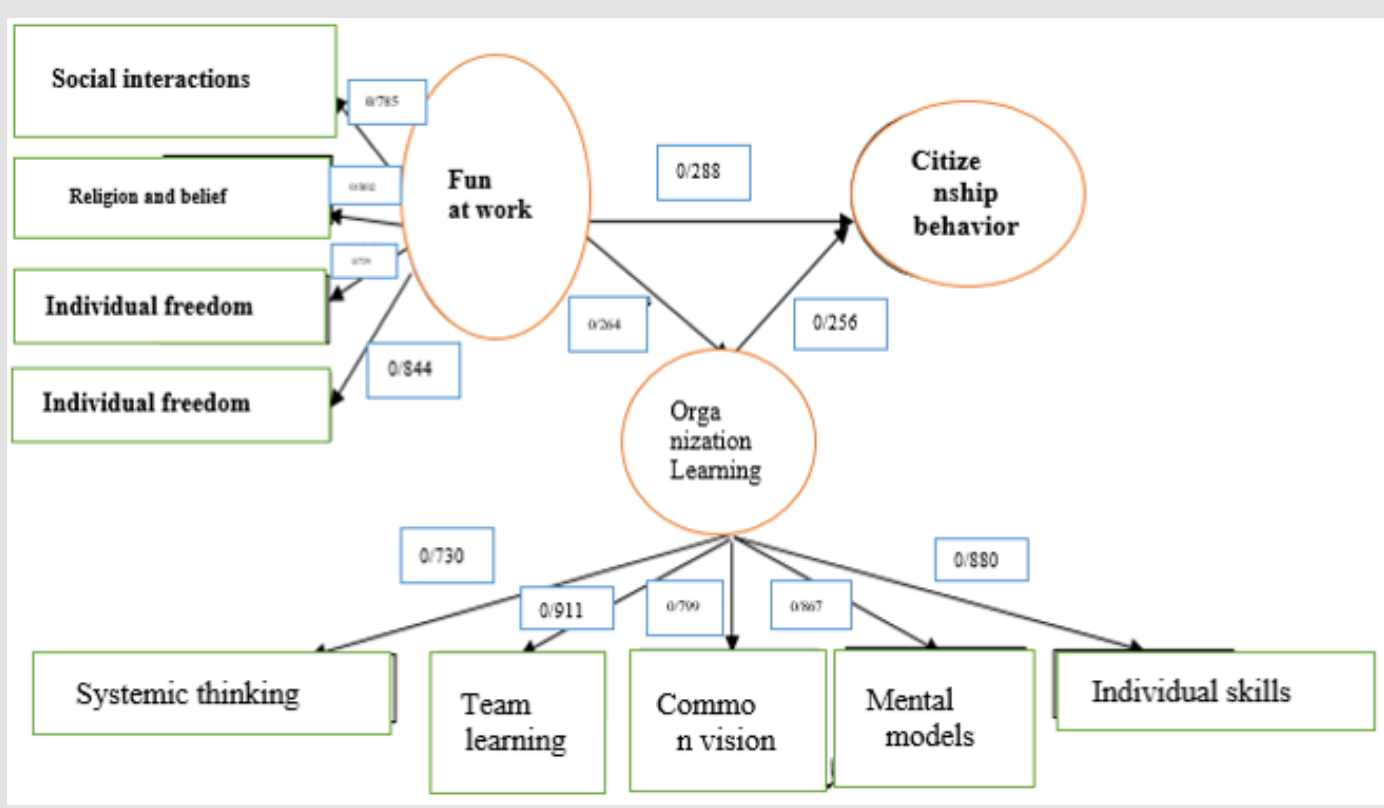

Figure 2: Measurement model.

1. Part related to measurement models,

2. Part related to structural model.

The reliability of the measurement model is evaluated by factor load coefficients, Cronbach's alpha and combined reliability. Factor load is calculated by calculating the correlation value of the indices of a structure with that structure. If this value is equal to or greater than the value of 0.4 , it confirms that the variance between the structure and its indices is greater than the variance of the measurement error of that structure. And reliability about that structure is acceptable. Content validity was also confirmed using CVI and CVR validity forms. The CVR value was 0.70 and the CVI value was more than 0.78 according to the number of professors (10 people). Therefore, the validity of the content of our tools was confirmed. Divergent and convergent validity related to structural equations were measured. In this study, two sections of descriptive statistics and inferential statistics were used. In the descriptive statistics section, descriptive tables and graphs were used to express the demographic and descriptive characteristics of the research. Use elongation and skewness indices to check the distribution of data (normal or abnormal) and to review and answer research questions and draw and develop a measurement model and structural model of SPSS software version 24 and Smart PLS version 3.0 (because the number of samples in the present study is less than 200 , this software was used).

\section{Findings}

The results of descriptive statistics on the statistical sample show that the highest age group of the research samples belonged to the age group. 30 to 40 years (52\%), 54\% were male and $46 \%$ were female. In terms of education, the highest number was related to the bachelor's degree group (55\%) and in terms of history, the highest group was related to the history of more than 15 years (42\%). According to Her et al. [27], skewness and elongation were used to determine the normality of the data. The results showed that the skewness is between 3 and 3 and the elongation is between 
5 and 5. Therefore, the data have a normal distribution. However, because one of the prerequisites for using covariance-based software is a sample of more than 200 people, and in this study, the number of samples is less than 200 people (173 people), so the use of covariance-based software is not allowed and variancebased software must be used. One of the most important variancebased software is Smart PLS, the latest version of which (No. 3) was used in this study. To evaluate the fit of measurement models, three criteria of reliability, convergent validity and divergent validity are used and their reliability is done through three factors: factor load coefficients, Cronbach's alpha coefficients, combined reliability and shared reliability. The value of the criterion for the appropriateness of factor load coefficients is 0.5 . For all items, the factor loading coefficients of the questions are greater than 0.5 , which indicates that this criterion is appropriate. None of the items were deleted here. The value of Cronbach's alpha and the combined reliability of the variables is higher than 0.7 , which indicates the appropriate reliability of the model. In this research, the reliability of the variables is at the desired level. Since the reliability is confirmed, the hypotheses can be examined by structural equations and the result can be generalized to the whole society. The second criterion for examining measurement models is convergent validity, which examines the correlation of each variable with its items (indicators).

According to the Fornell and Larker methods, which have introduced a suitable value for AVE of 0.4 and above. For all variables, the value of AVE is greater than or equal to 0.4. Divergent validity is the third criterion for examining the fit of measurement models. In this study, in both parts of the first method (questions related to each variable are more correlated with the variable itself than with other variables) and second (another important criterion with Divergent validity is determined, the degree of relationship of one variable with its questions compared to the relationship of that variable with other variables, so that the acceptable divergent validity of a model indicates that one variable in the model interacts more with questions than with other variables. Divergent validity was confirmed when it was acceptable when the AVE value for each variable was greater than the common variance between that variable and the other variables in the model. After examining the fit of the measurement models, it is time to fit the structural model of the research. As mentioned before, the structural model section, unlike measurement models, has nothing to do with questions (explicit variables) and only hidden variables with relationships between them are examined. To evaluate the fit of the structural model of the research, several criteria are used, the first and most basic of which are the significant coefficients $t$ or the values of $t$-values. If the value of these numbers exceeds 0.95 , it indicates the correctness of the relationship between the variables and thus confirms the research hypotheses at the confidence level of 1.96. Of course, it should be noted that numbers only show the correctness of the relationship and the intensity of the relationship between the variables cannot be measured by it.

The value of R2 for the dependent variable of organizational learning was 0.35 , which indicates its high value, and for the dependent variable. Organizational citizenship behavior was 0.49 , which again indicates its high level. Q2 value: (Table 1) This criterion determines the predictive power of the model and if the value of Q2 in the case of one of the endogenous variables acquires three values of $0.02,0.15$ and 0.32 , respectively, it indicates the power Has a weak, medium and strong prediction of the variable or related exogenous variables. This criterion for both endogenous variables is more than 0.32 , which indicates that the exogenous (independent) variables are strong in predicting the dependent variables and confirms the proper fit of the structural model of the research to some extent. Overall model fit: The overall model includes both the measurement and structural model parts, and by confirming its fit, the fit check in a complete model is completed. Given the three values of $0.01,0.35$ and 0.36 which are introduced as weak, medium and strong values for GOF. This criterion for both endogenous variables is more than 0.36 , which indicates a strong overall fit of the model. According to the table above, the value of T-Value is confirmed by the fact that all of them are more than 2.58 , so at the level of 0.99 , each of the relationships in the conceptual model of the present study is confirmed. In the following, the results of mediating the organizational learning variable are reported in (Table 2). According to Table 2, "Organizational learning" has a partial mediating role on the relationship of "fun at work" and "Citizenship behavior".

Table 1: Investigating the relationships of the research conceptual model.

\begin{tabular}{|c|c|c|}
\hline Independent Variable & The Dependent Variable & Multiply the Path \\
\hline Fun at work & Organisational Learning & $264 \%$ \\
\hline Fun at work & Citizenship behavior & $288 \%$ \\
\hline organisational Learning & Citizenship behavior & $2 / 893$ \\
\hline
\end{tabular}

Table 2: Investigating the effect of mediator variable on the relationships between variables.

\begin{tabular}{|c|c|c|c|c|}
\hline Mediator & Independent Variable & The Dependent Variable & Direct Impact & Indirect Effect \\
\hline $\begin{array}{c}\text { Organizational } \\
\text { Learning }\end{array}$ & Fun at work & Citizenship behavior & $288 \%$ & $140 \%$ \\
\hline
\end{tabular}




\section{Discussion}

The results showed that there is a significant relationship between fun atworkand organizational citizenship behavior with the mediating role of organizational learning among physical education teachers in Kermanshah course. This result is consistent with the results of research by Mikael, Geon Sick, Andres and Michael et al. [28-31] found that fun at work improves job performance. Plaster and Hatchi Sun (2016) argued that refreshment at work increases the work ethic. Geon Sik et al. [32] also found that refreshment at work has a significant effect on organizational commitment and job satisfaction of nurses. also found in their research that happiness and refreshment can improve organizational citizenship behavior and the ability of employees to learn. found in their research that there is a significant relationship between refreshment in work and organizational citizenship behavior. also found that refreshment at work has a direct and indirect effect on organizational citizenship behavior. However, more research is needed to understand the different contexts of this relationship and the role of mediators in this relationship. Confirming the results of this study, we can also refer to the study of Van Pierne (2003) which states that when employees are happy in their workplace, the relevant organization or company provides a better system of activity. Geon Sik et al. [32] also found that employees' sense of empowerment and happiness and the level of their organizational citizenship behaviors are related. Spindler et al. [30] believe that there is a relationship between positive emotion and happiness with the establishment of broad social relationships, helpful behaviors, accuracy, focus and high decision-making ability, which are consistent with the results of the present study. Geon Sik et al. [32] also encourages the work of increasing emotional commitment and emotional attachment to the organization, and people with emotional commitment to the organization are more likely to take action to benefit their organization and citizenship behaviors.

Therefore, in line with these results, by developing different dimensions of fun at work, especially the dimension of social interactions and rituals and holidays, can increase organizational citizenship behavior. The results also showed that organizational learning has a mediating role in the relationship between culture and citizenship behavior and is a partial mediation. Consistent with this conclusion, in their research found that refreshing in the organization increases learning in the organization. Found that there is a significant relationship between organizational learning and organizational citizenship behavior. also found that happiness and well-being can improve organizational citizenship behavior and the ability of employees to learn. Explaining the result of the present study on the mediating role of organizational learning in the relationship between recruitment at work and citizenship behavior, it can be said that organizational learning requires effective communication and recreation at work facilitates effective communication in the organization and Access to information and vital resources in order to improve performance and appropriate use of environmental opportunities and the emergence of such an environment can lead to the expansion of organizational citizenship behavior.

Also, excellence in work among group members leads to the formation of group personality and promotes cohesion and integration among members, which promotes mutual help and organizational citizenship behaviors among members. Based on what has been said, the development of fun at work in physical education teachers can directly and indirectly increase citizenship behavior. Therefore, it is suggested that managers and officials, in order to achieve employees with the ability to provide the best possible services, to develop a refreshing dimension in the work of physical education teachers, explaining that one of the ways to achieve this is to create space. Is to motivate employee participation in activities and actions beyond the role. For example, creating a friendly environment and an attractive and relaxing work environment (both physically and mentally) and the special attention of the managers of these offices to creating a fun environment that refers to the overall atmosphere of fun at work. It can create positive communication, interactions and create an atmosphere to increase organizational learning as well as stress reduction. These result in an increase in organizational citizenship behavior and performance improvement.

\section{References}

1. Ahmadi AS, Mobaraki H, yosufli M (2012) Structural Relationship between Job Satisfaction with Organizational Citizenship Behavior of Nurses in Educational Hospitals of Qazvin and Isfahan Provinces. Journal of Qazvin University of Medical Sciences 16 (2): 76-71.

2. Alegre J, Chiva R (2008) Assessing the impact of organizational learning capability on product innovation performance. Technovation 28(6): 315-326.

3. Baharifar A, Javaheri Kamel M, Ahmadi AA (2011) "Ethical Behavior and Organizational Citizenship Behavior: The Impact of Ethical Values, Justice and Organizational Commitment". Research on Organizational Resource Management, 1(1): 0-0.

4. Cheung MF Y (2013) The mediating role of perceived organizational support in the effects of interpersonal and informational justice on organizational citizenship behaviors. Leadership \& Organization Development Journal 34(6): 551-572.

5. Chi-Chang C, Meng-Chen T, Meng-Shan T (2011) The organizational citizenship behaviors and organizational commitments of organizational members influences the effects of organizational learning. International Journal of Trade, Economics and Finance 2(1): 61-66.

6. Fluegge W (2014) Play hard, work hard, Management Research Review 37(8): 682-705.

7. Foot DA, Li-Ping Tang $T$ (2008) Job satisfaction and organizational citizenship behavior OCB: Does team commitment make a difference in self-directed teams. Management Decision 46(6): 933-947.

8. Geum Sik Ahn, Seewon Ryu (2017) Effects of Workplace Fun to Job Satisfaction and Work Engagement of Hospital Nurses. Journal of Health Informatics and Statistics. J Health Info Stat 42(2): 144-152.

9. Golparvar M, Padash F, Firepour H (2010) The model enhances the sense of energy, empowerment and creativity of employees through ethical leadership. Quarterly Journal of Health in Iran. (3): 4-11. 
10. Huang JH, Jin BH, Yang C (2004) Satisfaction with business to- employee benefit systems and organizational citizenship behavior $\gg$, International Journal of Manpower 25(2).

11. Javaheri Kamel M (2009) Organizational Justice, The Relationship of Leader-Member, Trust and Psychological Empowerment on Organizational Citizenship Behavior, Policeman Human Development Bulletin, 24: 21-27.

12. Karatepe OM (2011) Perceptions of organizational politics and hotel employee Outcomes: The mediating role of work engagement. International Journal of Contemporary Hospitality Management 25(1): 82-10.

13. Karl KA, Peluchette JV, Harland L (2007) Is fun for everyone? Personality differences in healthcare providers attitudes toward fun. Journal of Health \& Human Services Administration 29(4): 409-447.

14. Lion MY, Mario F, Li-Ping Tang T (2011) Self-defined effect on the relationship between organizational citizenship behavior and perceived organizational justice. Leadership \& Organization Development Journal 34(6): 532- 550.

15. McDowell (2004) Fun at work: Scale development, confirmatory factor analysis, and links to organizational outcomes. unpublished Ph.D, Alliant International University, united states, San Diego.

16. Michael J, Tews JW, Noe RA (2017) Does fun promote learning? The relationship between fun in the workplace and informal learning. 98 46-55.

17. LiangGuo (2018) A social exchange perspective on why and when ethical leadership foster customer-oriented citizenship behavior. International Journal of Hospitality Management 70: 1-8.

18. Organ DW (1990) The motivational basis of organizational citizenship behavior, Research in Organizational Behavior 3(12): 43-72.

19. Petrou, Anastasia, Daskalopoulou Irene (2013) Social capital and innovation in the services sector. European Journal of Innovation Management 16(1): 50-69.

20. Plester B, Hutchison A (2016) "Fun times: the relationship between fun and workplace engagement." Employee Relations 38(3): 332-350.

21. Podsakoff, Mackenzie, paine, Bachrach (2000) "organizational citizenship behaviours: a critical review or the theoretical and empirical literature and suggestions for future research", Journal of Management 26(3): 513-563.

ISSN: 2574-1241

DOI: 10.26717/BJSTR.2021.33.005332

Mohammad Saeid Kiani. Biomed J Sci \& Tech Res

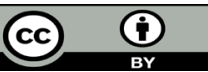

This work is licensed under Creative Commons Attribution 4.0 License

Submission Link: https://biomedres.us/submit-manuscript.php
22. Rahimnia F, Sirih N (2017) The role of mediators among staff members in the relationship between organizational excellence and organizational citizenship and behavior. Journal of Management Studies (Improvement and Development). Years Twenty-Six 84: 32-41.

23. Robbins, Stefan P, Jayge, Timothy (2009) Organizational Behavior, Vol. 1 , Translation by Mehdi Zare, Tehran: Nasr:45.

24. Shane Pe, LeelaRaja, Brienna Andrews, Julia Lui (2018) Perceptions of organizational culture and organizational citizenship by faculty in U.S. colleges and schools of pharmacy. Currents in Pharmacy Teaching and Learning. Available online 10(4): 403-412

25. Shiri Lavy, Hadassah Littman-Ovadia (2017) My better self: using strengths at work and work productivity, organizational citizenship behavior, and satisfaction. Journal of Career Development 44(2): 21-30.

26. Sohrabi S, Alavi M (2010) Citizenship Behavior, Business Management 8 (2): 171-182

27. Soleimani E, Ahanchian, Asghar pour massoule MR (2013) The role of organizational identity in predicting organizational citizenship behavior in Razavi Islamic University of Mashhad. Social Sciences Social.

28. Somech A, Drach Zahavy A (2004) Exploring organizational citizenship behavior from an organizational learning and organizational citizenship behavior. Journal of Occupational and Organizational Psychology 77(3): 281-298.

29. Spindler, Kruse C, Pederson, SS (2009) Positive affect and negative affect correlate differently with distress and health-related quality of life in patients with cardiac conditions: Validation of the Danish global mood scale. Journal of Psychosomatic Research 67 (1): 57-65.

30. Uzma Quraishi, Fakhra Aziz, Tanya Vaughan (2018) An Investigation of Authentic Leadership and Teachers 'Organizational Citizenship Behavior in Secondary Schools of Pakistan.

31. Yilmaz ML, Tasdan O (2008) Relationship between organizational justice and organizational citizenship behaviors primary teachers. Management Decision 46(6): 933-947.

32. Yilmez Yursad, Tasdon Murat (2009) Organizational Citizenship and Organizational Justice in Turkish Primary Schools. Journal of Educational Administration 47(1): 108-126.

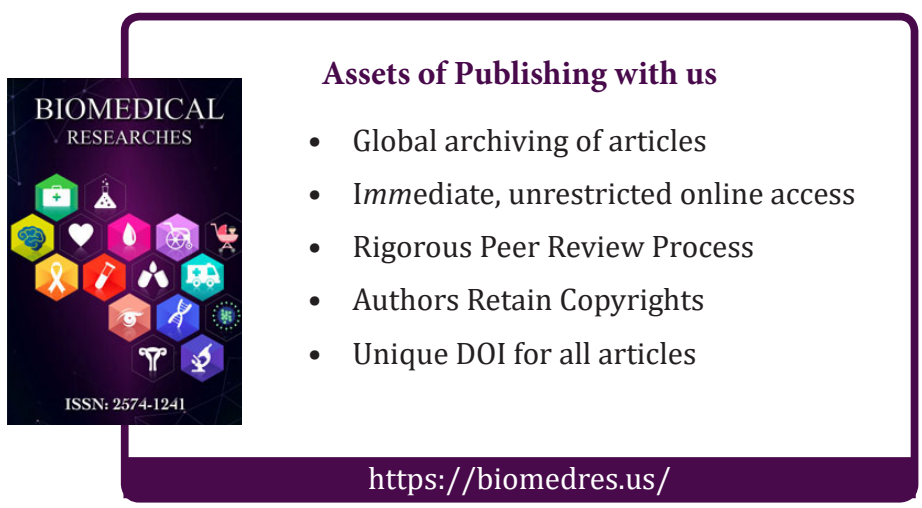

Copyright@ Mohammad Saeid Kiani | Biomed J Sci \& Tech Res | BJSTR. MS.ID.005332. 